\title{
Reduction of inflammation after administration of interleukin-1 receptor antagonist following aneurysmal subarachnoid hemorrhage: results of the Subcutaneous Interleukin-1Ra in SAH (SCIL-SAH) study
}

\author{
James Galea, PhD, ${ }^{1,2}$ Kayode Ogungbenro, PhD, ${ }^{3}$ Sharon Hulme, ${ }^{2}$ Hiren Patel, PhD, ${ }^{2}$ \\ Sylvia Scarth, BSc, ${ }^{2}$ Margaret Hoadley, PhD, ${ }^{2}$ Karen Illingworth, MSc, ${ }^{2}$ \\ Catherine J. McMahon, PhD, ${ }^{4}$ Nikolaos Tzerakis, FRCS, ${ }^{5}$ Andrew T. King, FRCS, ${ }^{2}$ Andy Vail, MSc, ${ }^{6}$ \\ Stephen J. Hopkins, PhD, ${ }^{2}$ Nancy Rothwell, FRS, ${ }^{7}$ and Pippa Tyrrell, FRCP ${ }^{2}$

\begin{abstract}
${ }^{1}$ Ninewells Hospital and Medical School, University of Dundee; ${ }^{2}$ Institute of Cardiovascular Sciences, Manchester Academic Health Sciences Centre, Salford; ${ }^{3}$ Centre for Applied Pharmacokinetic Research, Manchester Pharmacy School; ${ }^{6}$ Centre for Biostatistics, Institution of Population Health, University of Manchester; ${ }^{7}$ Faculty of Life Sciences, University of Manchester; ${ }^{4}$ Walton Centre for Neurology and Neurosurgery, Fazakerley, Liverpool; and 5 University Hospital of North Midlands, Royal Stoke University Hospital, Stoke-on-Trent, United Kingdom
\end{abstract}

\begin{abstract}
OBJECTIVE Aneurysmal subarachnoid hemorrhage (aSAH) is a devastating cerebrovascular event with long-term morbidity and mortality. Patients who survive the initial bleeding are likely to suffer further early brain injury arising from a plethora of pathological processes. These may result in a worsening of outcome or death in approximately $25 \%$ of patients and may contribute to longer-term cognitive dysfunction in survivors. Inflammation, mediated by the cytokine interleukin-1 (IL-1), is an important contributor to cerebral ischemia after diverse forms of brain injury, including aSAH. Its effects are attenuated by its naturally occurring antagonist, IL-1 receptor antagonist (IL-1Ra [anakinra]). The authors hypothesized that administration of additional subcutaneous IL-1Ra would reduce inflammation and associated plasma markers associated with poor outcome following aSAH.
\end{abstract}

METHODS This was a randomized, open-label, single-blinded study of $100 \mathrm{mg}$ subcutaneous IL-1Ra, administered twice daily in patients with aSAH, starting within 3 days of ictus and continuing until 21 days postictus or discharge from the neurosurgical center, whichever was earlier. Blood samples were taken at admission (baseline) and at Days 3-8, 14 , and 21 postictus for measurement of inflammatory markers. The primary outcome was difference in plasma IL-6 measured as area under the curve between Days 3 and 8 , corrected for baseline value. Secondary outcome measures included similar area under the curve analyses for other inflammatory markers, plasma pharmacokinetics for IL-1Ra, and clinical outcome at 6 months.

RESULTS Interleukin-1Ra significantly reduced levels of IL-6 and C-reactive protein $(p<0.001)$. Fibrinogen levels were also reduced in the active arm of the study $(p<0.002)$. Subcutaneous IL-1Ra was safe, well tolerated, and had a predictable plasma pharmacokinetic profile. Although the study was not powered to investigate clinical effect, scores of the Glasgow Outcome Scale-extended at 6 months were better in the active group; however, this outcome did not reach statistical significance.

CONCLUSIONS Subcutaneous IL-1Ra is safe and well tolerated in aSAH. It is effective in reducing peripheral inflammation. These data support a Phase III study investigating the effect of IL-1Ra on outcome following aSAH.

Clinical trial registration no.: EudraCT: 2011-001855-35 (www.clinicaltrialsregister.eu)

https://thejns.org/doi/abs/10.3171/2016.9.JNS16615

KEY WORDS neuroinflammation; aneurysmal subarachnoid hemorrhage; interleukin-1 receptor antagonist; interleukin-6; C-reactive protein; CRP; fibrinogen; vascular disorders; inflammation; Phase II trial

\footnotetext{
ABBREVIATIONS AE = adverse event; aSAH = aneurysmal subarachnoid hemorrhage; $A U C=$ area under the curve; $C R P=C$-reactive protein; GOS-E = Glasgow Outcome Scale-extended; IL = interleukin; IL-1Ra = IL-1 receptor antagonist; IMP = investigational medicinal product; IV = intravenous; In = natural log; SAE = serious AE; SC = subcutaneous; WFNS $=$ World Federation of Neurosurgical Societies
}

SUBMITTED March 24, 2016. ACCEPTED September 22, 2016.

INCLUDE WHEN CITING Published online February 24, 2017; DOI: 10.3171/2016.9.JNS16615. 
A NEURYSMAL SAH (aSAH) is an acute cerebrovascular event characterized by the rupture of an aneurysm within the subarachnoid space. There is initial global and local cerebral ischemia that constitutes the first insult. Subsequent secondary brain injury is multifactorial in origin, with vasospasm, brain edema, and cerebral inflammation thought to be major contributors. It affects $30 \%-50 \%$ of survivors and results in a high morbidity and mortality. ${ }^{8}$ In addition, the long-term effects of aSAH in terms of cognitive and higher mental function are increasingly recognized to cause a significant lifelong burden. ${ }^{28}$

There is evidence that a significant inflammatory response is seen in patients following all acute cerebrovascular events, in particular aSAH. ${ }^{24}$ Interleukin (IL)-1 is a proinflammatory cytokine that is an important mediator of inflammation-mediated neuronal damage. IL-1 and its downstream mediators (in particular IL-6) are not neurotoxic in the normal milieu, but become significantly deleterious in neuropathological states such as intracerebral bleeding. In preclinical studies, IL-1 has been demonstrated to alter blood-brain barrier permeability ${ }^{5}$ and to induce glial-mediated neurotoxicity. ${ }^{34}$ Neutralization of IL-1 using IL-1 $\beta$ antibodies reduces vasospasm in experimental $\mathrm{aSAH}^{19}$ that leads to exacerbation of preexisting cerebral ischemia.

Clinical observational studies have demonstrated that high concentrations of IL-1 and IL- 6 are associated with vasospasm following SAH. ${ }^{18,27,30}$ Recent studies using microdialysis technology have demonstrated that IL-6 is associated with incidence of vasospasm as well as a poor functional outcome following aSAH. ${ }^{16}$ In addition, there is emerging evidence that following hemorrhagic stroke, hemoglobin and its breakdown products act as damageassociated molecular patterns and elicit an IL-1-mediated sterile inflammatory response that is neurotoxic. ${ }^{13}$

Interleukin-1 receptor antagonist (IL-1Ra) is the naturally occurring antagonist to IL-1. It has been proven to be neuroprotective in various models of cerebral ischemia, including ischemic stroke. ${ }^{4}$ Anakinra, a recombinant form of IL-1Ra, is used clinically as a second-line drug for autoimmune diseases such as rheumatoid arthritis and is currently being investigated in $>40$ clinical conditions. Its safety profile in acute neurodegenerative disease and aSAH in particular has been well established., ${ }^{6,11,32}$ Peripherally administered anakinra has been demonstrated to cross the blood-CSF barrier and the blood-brain barrier after SAH and traumatic brain injury. ${ }^{14,17}$ The biological activity and pharmacodynamics of subcutaneous (SC) IL$1 \mathrm{Ra}$ have been studied extensively. ${ }^{3}$

We previously demonstrated that intravenous (IV) anakinra reduces plasma and CSF IL-6 concentrations in patients with aSAH. ${ }^{32}$ Intravenous administration is not as practical or as cost-effective as SC delivery for long-term administration during the period of heightened risk from secondary brain injury. The aim of the present study was to investigate the effect of SC IL-1Ra on the peripheral inflammatory response following aSAH, as a proof of concept of its biological effect in this patient population. We tested the feasibility and tolerability of twice-daily SC administration of IL-1Ra and obtained early data on efficacy to inform the design of future Phase III studies.

\section{Methods}

\section{Overall Study Design}

The Subcutaneous Interleukin-1Ra in SAH (SCILSAH) study was a 3-center, open-label, randomized controlled Phase II trial. Patients were recruited over a 36-month period (November 2011 to November 2014) from 3 major neurosciences centers in the North of England: Salford Royal Foundation Trust, The Walton Neurological Centre, and University Hospital North Staffordshire. Approvals were obtained from the National Research Ethics Committee (11/NW/0390) and the UK Medicine and Healthcare products Regulatory Agency (MHRA) (European Union Clinical Trials Register, https://www. clinicaltrialsregister.eu, EudraCT: 2011-001855-35).

\section{Eligibility and Screening}

Participants were considered eligible for the study if they had radiologically confirmed aSAH and if consent could be obtained and drug administered within 72 hours of ictus. Patients were excluded if they were $<18$ years old, had abnormal renal function (creatinine $>177 \mu \mathrm{mol} / \mathrm{L}$ ), had a previously recorded allergy to Escherichia coli, had known or suspected infection in the preceding 2 weeks or at the time of the study, or had participated in a clinical trial of an investigational medicinal product (IMP) in the preceding 30 days. Pregnant or breastfeeding patients, as well as those with active malignancy, were excluded. Verbal and written information was provided by the research team and a reapproach was made for consent from the patient or their legal representative. For those patients who were not able to provide initial consent but who regained capacity during the course of the study, reconsent was sought and obtained.

\section{Randomization and Masking}

As soon as consent was obtained, a baseline assessment was performed on each participant and a blood sample of up to $20 \mathrm{ml}$ was taken. Clinical assessment included standard measures of aSAH severity, including the Fisher grade $^{9}$ and World Federation of Neurosurgical Societies (WFNS) grade. ${ }^{33}$ Randomization to treatment or no treatment (1:1) was stratified for Day 1, 2, or 3 postictus. A second randomization allocated patients into 1 of the 3 plasma-sampling time groups (07:00, 13:00, or 19:00 hours), stratified by their allocated intervention group. This block sampling technique was designed to allow us to obtain 3 samples per day to confirm the pharmacokinetics of our chosen regimen without the need for repeated venipuncture of individuals. The process was performed using an Internet-based third-party randomization service (www. sealedenvelope.com), and the investigators were unaware of the block size throughout the study period. The control and IMP arms of the study were not matched.

\section{Intervention Procedures}

For participants allocated to the IMP arm, SC anakinra was injected at the next administration time point (07:00 or 19:00 hours) \pm 2 hours, and continued twice daily until a 


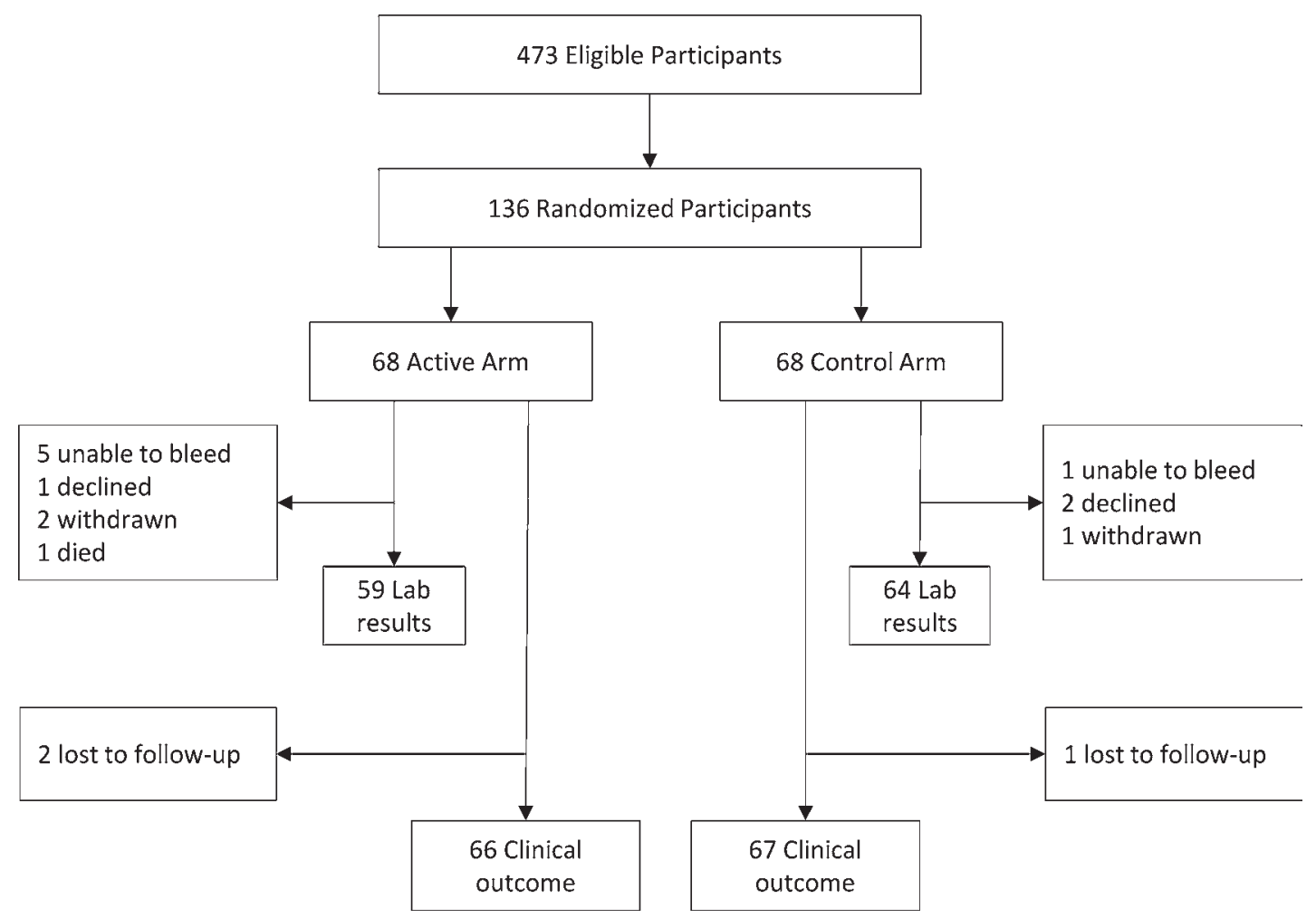

FIG. 1. Screening and recruitment schema.

maximum of 21 days postictus or discharge from neurosurgical care. This dosing regimen was informed by a pharmacokinetic model and tolerability data obtained from Amgen, Inc. It reflected a compromise between a relatively steady plasma concentration, our mean target concentration, and an acceptable rate of injection-site reactions. All patients underwent attempts at blood sampling daily from Days 3 to 8 , and then on Days 14 and 21 postictus for those who were still inpatients at the neuroscience center.

Optimization of sampling times was performed using PopDes, as published previously. ${ }^{15}$ All adverse events (AEs) were classified in line with published MHRA definitions (http://www.hra.nhs.uk/documents/2015/06/ safety-progress-reports-procedural-table-ctimps.pdf) and were reported in all patients up to 30 days from cessation of therapy (per Good Clinical Practice) to the independent data monitoring and safety committee. Patients were monitored closely; those with evidence of neurological deterioration underwent angiography to exclude symptomatic vasospasm, per our routine clinical practice.

\section{Sample Processing and Analysis}

Blood samples were collected into tubes containing EDTA and centrifuged at $2000 \mathrm{~g}$ at $4^{\circ} \mathrm{C}$ for 15 minutes. Plasma was frozen at $-70^{\circ} \mathrm{C}$. All samples were transported to Salford Royal Foundation Trust and batch analyzed. Concentrations of IL-6, IL-8, and C-reactive protein (CRP) were measured using Luminex bead technology. Interleukin-1Ra was measured by enzyme-linked immunosorbent assay and fibrinogen was measured by functional assay. Laboratory staff were blinded to treatment allocation, except for the individual measuring IL-1Ra.

\section{Statistical Analysis}

The primary outcome measure was the area under the curve (AUC) for natural log (ln) (IL-6) from Days 3 to 8. Patients were included in the analysis if they provided $\geq 4$ blood samples from a possible total of 6 . Secondary outcomes were corresponding AUCs for other biomarkers, all biomarkers up to Day 21, and Glasgow Outcome Scale-extended (GOS-E) score at 6 months. Sample size calculation was based on effects observed for $\ln (\mathrm{IL}-6)$ and $\ln (\mathrm{CRP})$ after 72 hours of IV infusion in patients with ischemic stroke in our previous trial. ${ }^{7}$ Our target recruitment was 64 participants per group for $80 \%$ power to detect a standardized effect size of 0.5. Analyses were on an intention-to-treat basis.

To calculate AUC, missing data points for an individual were imputed by fitting a linear regression to observed values on the log scale. Analysis of covariance was used to estimate the group difference for inflammatory markers, adjusting for baseline values of the relevant marker. The GOS-E scores were compared using ordinal logistic regression after confirming the proportional odds assumption. All analyses were adjusted for the randomization strata. Concentrations of IL-1Ra were plotted against prediction intervals calculated using the population pharmacokinetic data a priori.

\section{Results}

\section{Patient Recruitment and Feasibility}

Four hundred seventy-three patients were screened, and 136 were recruited from the 3 centers between October 2011 and November 2014 (Fig. 1, Table 1). Thirteen 
TABLE 1. Demographic data and clinical characteristics for study participants in the Subcutaneous Interleukin-1Ra (SCIL) study

\begin{tabular}{ccc}
\hline \multicolumn{1}{c}{ Characteristic } & $\begin{array}{c}\text { Active Group, } \\
n=68, \text { No. }(\%)\end{array}$ & $\begin{array}{c}\text { Control Group, } \\
\mathrm{n}=68, \text { No. }(\%)\end{array}$ \\
\hline Center & & \\
\hline Salford & $57(84)$ & $55(81)$ \\
\hline Walton & $5(7)$ & $8(12)$ \\
\hline Stoke & $6(9)$ & $5(7)$ \\
\hline Mean age in yrs, range & $52,24-77$ & $52,23-74$ \\
\hline Sex, M & $12(18)$ & $18(26)$ \\
\hline Smoking status & & $18(26)$ \\
\hline Nonsmoker & $24(35)$ & $15(22)$ \\
\hline Former smoker & $7(10)$ & $35(51)$ \\
\hline WFNS grade & & $19(28)$ \\
\hline I & $37(54)$ & $2(3)$ \\
\hline II & $14(21)$ & $8(12)$ \\
\hline III & $4(6)$ & $4(6)$ \\
\hline IV & $10(15)$ & $1(1)$ \\
\hline V & $3(4)$ & $8(12)$ \\
\hline Fisher grade & & $22(32)$ \\
\hline 1 & $1(1)$ & \\
\hline 2 & $5(7)$ & \\
\hline 3 & $18(26)$ & \\
\hline 4 & $44(65)$ & \\
\hline
\end{tabular}

participants $(10 \%)$ withdrew or were withdrawn from blood sampling, including 3 who became severely ill and were not expected to survive; 3 who were withdrawn for medical reasons (elevated liver function tests and rash of unknown etiology); and 2 who withdrew because of injection-site reactions. Patients ranged in age from 23 to 77 years, with a median age of 52 years. More than $80 \%$ had Fisher Grade 3 or 4, and a similar proportion had WFNS Grade I or II.

\section{Pharmacokinetic Profiles}

The plasma pharmacokinetic profile of IL-1Ra in patients receiving anakinra was within our predicted intervals (Fig. 2), although the observed mean concentrations were lower than the predicted mean. Twice-daily dosing achieved a steady concentration profile with minimal peak trough fluctuation. The maximum plasma concentration was $1.6 \mu \mathrm{g} / \mathrm{ml}$, and the minimum concentration was above our target median inhibitory concentration of $100 \mathrm{ng} / \mathrm{ml}$.

\section{Laboratory Results of Inflammatory Markers}

Circulating IL-1Ra concentrations confirmed that all participants had been treated as allocated. Concentrations of IL-6 were elevated in both groups at baseline, remaining high in untreated participants, but falling by Day 3 postictus in IL-1Ra-treated participants. The mean values in untreated patients began to decline at approximately 1 week and continued to decline in both groups up to 3

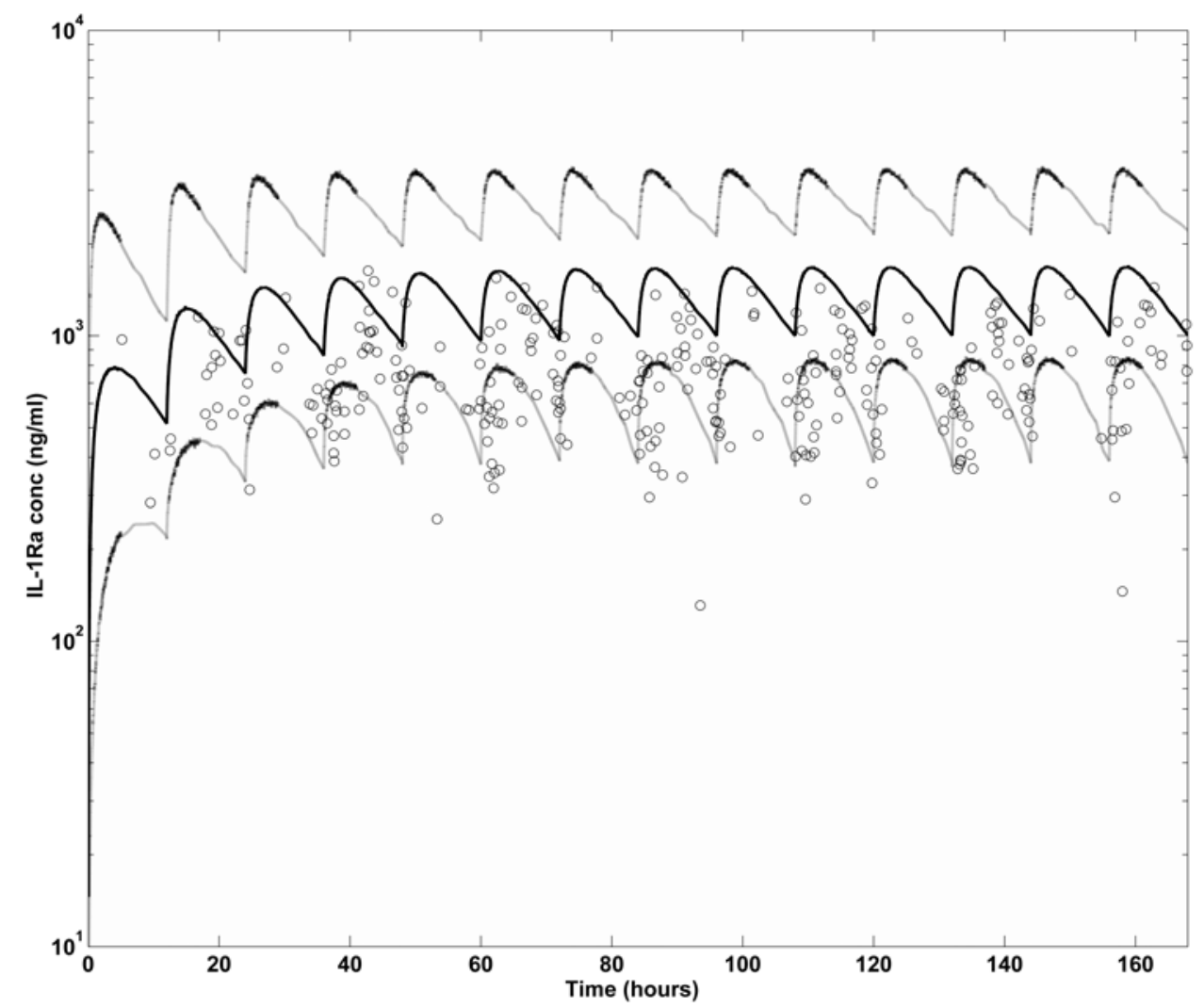

FIG. 2. Predicted (mean value in solid line, prediction intervals in dashed lines) and observed (dots) concentrations of IL-1Ra in patients receiving anakinra. Observed concentrations of IL-1Ra were within our predicted intervals, although mean values were below those predicted. conc $=$ concentration . 

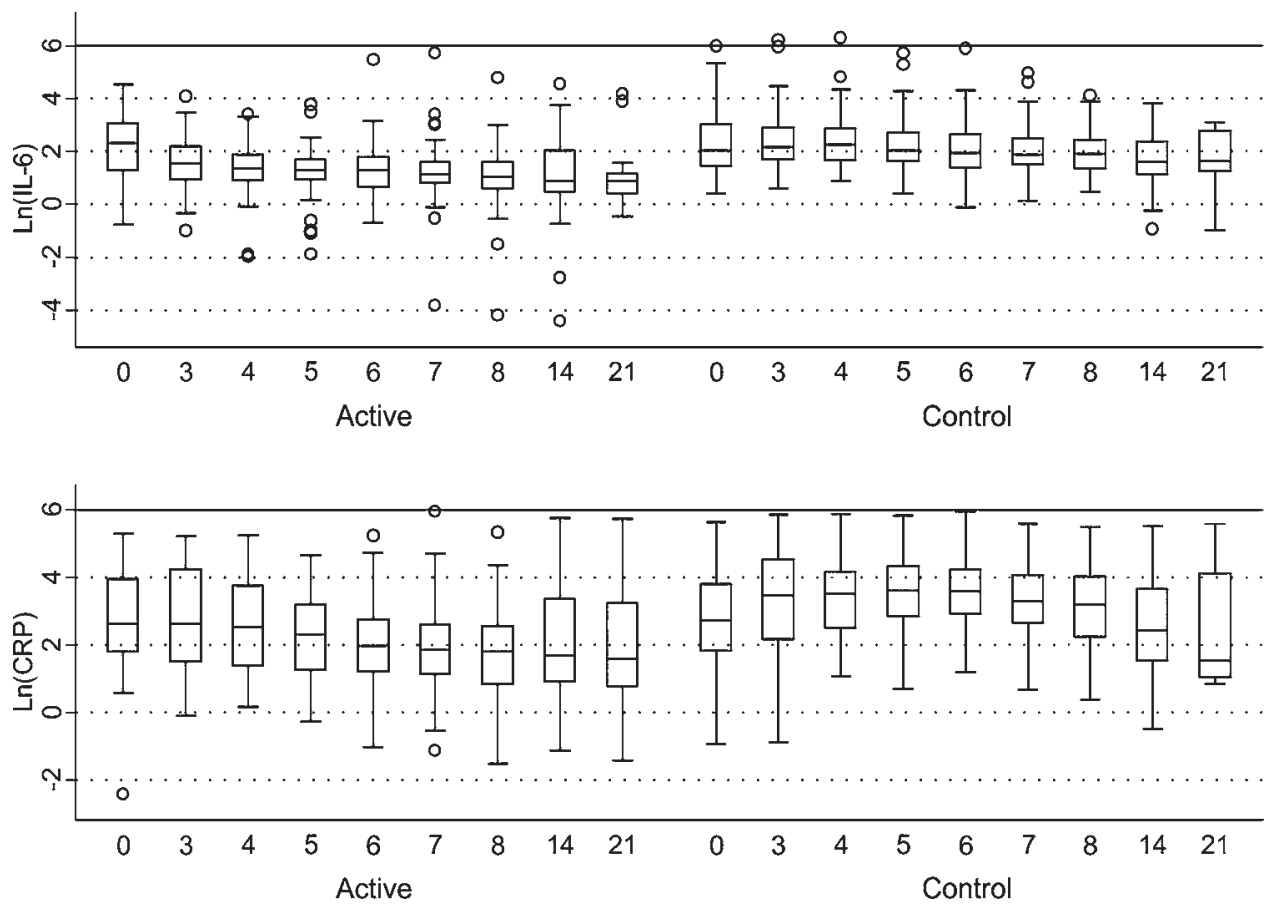

FIG. 3. Upper: $\ln (\mathrm{IL}-6)$ for patients receiving anakinra and control subjects at different time points. Lower: $\ln (\mathrm{CRP})$ for patients receiving anakinra and control subjects at different time points.

weeks (Fig. 3 upper). The mean levels of CRP showed a similar pattern of decline in each group, but decline was delayed by a few days in the control group (Fig. 3 lower). Fibrinogen levels remained stable in treated participants but increased over the 1st week in control participants (data not shown). Levels of IL-8 remained stable throughout the sampling period. There were statistically significant differences $(\mathrm{p}<0.001)$ between allocated groups for AUC analyses of both IL-6 and CRP (Table 2). Sensitivity analyses for method of imputation made no substantive difference to the conclusions. There was also a statistically significant difference in fibrinogen levels $(p=0.002)$ but no difference in IL-8 levels between the 2 groups.

\section{Clinical Outcome}

Three patients were lost to follow-up. Two of these patients returned overseas and could not be contacted. The third patient was uncontactable, but his clinical review notes reported "permanent neurological deficit, due to memory problems and lethargy." The GOS-E scores (a secondary outcome measure) were better in the active than in the control group, although this was not statistically significant (OR 0.92, 95\% CI 0.50-1.7; $\mathrm{p}=0.80$ [Table 3]).

\section{Safety and AEs}

The AEs and serious AEs (SAEs) are listed in Table 4. None of the SAEs were attributable to the IMP. Ten of 62 patients $(16 \%)$ in the IMP group experienced an injectionsite reaction. Forty-six infections were detected in our 2 cohorts. There were no differences between the groups, either in the incidence of overall infections (i.e., those classified as an AE or an SAE) (OR 0.88, 95\% CI 0.43-1.8) or of those infections classified as an SAE (i.e., the more severe ones) (OR 1.5, 95\% CI 0.62-3.7). The overall incidence of delayed cerebral ischemia (DCI) was 42 patients (31\%), of whom $22(35 \%)$ were in the treatment group and 20 (32\%) were in the control group. Cerebral vasospasm was demonstrated angiographically in 14 patients $(23 \%)$ and 16 patients $(26 \%)$ in these groups, respectively.

\section{Discussion}

This Phase II study demonstrates that recombinant IL1Ra, by SC administration, suppresses the IL-1-mediated response following aSAH. Inflammation mediated by IL-1 is increasingly recognized as an important contributor to neurodegeneration after acute cerebrovascular events. Al-

TABLE 2. The mean plasma concentrations of IL-6, CRP, IL-8, and fibrinogen in active and control participants

\begin{tabular}{lcccc}
\hline \multicolumn{1}{c}{ Variable } & Active Group, Mean (SD) & Control Group, Mean (SD) & Difference, Mean (95\% Cl) & $p$ Value \\
\hline $\ln ($ IL-6) & $6.37(4.25)$ & $11.04(4.02)$ & $4.48(3.28$ to 5.68$)$ & $<0.001$ \\
\hline $\ln ($ CRP) & $11.02(6.21)$ & $17.02(5.44)$ & $6.13(4.61$ to 7.66$)$ & $<0.001$ \\
\hline $\ln ($ IL-8) & $13.45(2.44)$ & $12.63(3.67)$ & $-0.31(-1.34$ to 0.72$)$ & 0.55 \\
\hline In(fibrinogen) & $7.61(1.87)$ & $8.59(1.71)$ & $0.97(0.36$ to 1.59$)$ & 0.002 \\
\hline
\end{tabular}

Includes values for both modeled and scaled methods for imputation of missing data. 
TABLE 3. Clinical outcomes of patients in active and control groups

\begin{tabular}{lcc}
\hline \multicolumn{1}{c}{$\begin{array}{c}\text { GOS-E Status } \\
\text { at } 6 \text { Mos }\end{array}$} & $\begin{array}{c}\text { Active Group, } \\
\mathrm{n}=66, \text { No. }(\%)\end{array}$ & $\begin{array}{c}\text { Control Group, } \\
\mathrm{n}=67, \text { No. }(\%)\end{array}$ \\
\hline Dead & $6(9)$ & $6(9)$ \\
\hline Persistent vegetative state & $0(0)$ & $1(1)$ \\
\hline Lower severe disability & $5(8)$ & $8(12)$ \\
\hline Upper severe disability & $12(18)$ & $6(9)$ \\
\hline Lower moderate disability & $1(2)$ & $0(0)$ \\
\hline Upper moderate disability & $6(9)$ & $9(13)$ \\
\hline Lower good recovery & $9(14)$ & $12(18)$ \\
\hline Upper good recovery & $27(41)$ & $25(37)$ \\
\hline
\end{tabular}

Estimated common OR $0.92,95 \% \mathrm{Cl} 0.50-1.7 ; p=0.80$.

though levels of both IL-1 and IL-6 are barely detectable in the brain under normal conditions, they can become rapidly upregulated to reach high concentrations, particularly in aSAH. Production of IL-6 can be upregulated by a wide variety of stimuli, including IL-1, tumor necrosis factor alpha, and transforming growth factor beta. High concentrations of IL-6 in plasma, CSF, and brain extracellular fluid are associated with poor outcome following SAH. $20,30,37$

We demonstrated previously that IL-1 antagonism using IV anakinra is a safe and effective way to reduce IL-6 following cerebral ischemic stroke ${ }^{7}$ and SAH..$^{32}$ In this study, we have proven that administration of SC IL-1Ra, starting within 72 hours and continued for a maximum period of 21 days postictus, is safe and significantly reduces plasma concentrations of IL- 6 following aSAH. This effect was seen as early as Day 3 postictus and persisted until Day 8 . Levels of CRP were also reduced in the active treatment group, but the reduction occurred more slowly than the reduction in IL-6. This would be in keeping with a rise in CRP, mediated partly by IL-6. C-reactive protein has been demonstrated to be an independent predictor of outcome for patients presenting with a good grade of SAH. ${ }^{36}$ Our results are encouraging because, in contrast to other drug trials, ${ }^{21}$ anakinra has significantly reduced CRP levels.

We did not detect any significant change in IL-8 levels in our study. Data from our previous preclinical studies have suggested that one of the mechanisms by which inflammation is detrimental to cerebral ischemia is through IL-1-induced potentiation of neutrophil mobilization via CXC chemokine induction. ${ }^{23}$ The IL-8 results in this study may have multiple implications. First, the mean IL-8 levels $(13 \mathrm{pg} / \mathrm{ml})$ were higher than those reported in a study of ischemic stroke $(4.3 \mathrm{pg} / \mathrm{ml}),{ }^{22}$ which might imply that neutrophil chemotaxis plays a more prominent role in aSAH. Second, although IL-1 can potentially upregulate both IL-6 and IL-8, it induces IL-6 gene expression in a mechanism independent of IL-8 upregulation, a phenomenon previously reported. ${ }^{29}$ Finally, IL-8 production may not be IL-1 dependent in aSAH. We have previously shown that antagonism of IL-1, using IL-1Ra, reduced neutrophil counts in patients with aSAH. ${ }^{11}$ However, it is also possible that either the dose of anakinra used in the current study was not sufficiently high to suppress IL-8-mediated neu-
TABLE 4. Numbers of SAEs and AEs reported for participants in active and control groups

\begin{tabular}{|c|c|c|c|c|}
\hline \multirow[b]{2}{*}{$\begin{array}{l}\text { Event } \\
\text { Type }\end{array}$} & \multicolumn{2}{|c|}{ No. of SAEs } & \multicolumn{2}{|c|}{ No. of AEs } \\
\hline & $\begin{array}{l}\text { Active } \\
\text { Group }\end{array}$ & $\begin{array}{l}\text { Control } \\
\text { Group }\end{array}$ & $\begin{array}{l}\text { Active } \\
\text { Group }\end{array}$ & $\begin{array}{l}\text { Control } \\
\text { Group }\end{array}$ \\
\hline Neurological & 25 & 24 & 12 & 7 \\
\hline Pyrexia of unknown origin & 3 & 1 & 9 & 11 \\
\hline \multicolumn{5}{|l|}{ Infection } \\
\hline Respiratory & 8 & 7 & 2 & 4 \\
\hline Urinary tract & 4 & 0 & 4 & 9 \\
\hline CNS & 2 & 3 & 0 & 0 \\
\hline Skin & 0 & 0 & 1 & 2 \\
\hline Metabolic & 1 & 1 & 7 & 14 \\
\hline Cardiac & 2 & 2 & 3 & 2 \\
\hline Other & 4 & 1 & 9 & 6 \\
\hline
\end{tabular}

trophilia or that IL-1Ra blocks polymorphonuclear mobilization independent of IL-8.

Fibrinogen levels were reduced in the active treatment group. This would be in keeping with the action of IL$1 \mathrm{Ra}$ in other inflammatory disease states, such as juvenile rheumatoid arthritis, where anakinra has an established therapeutic use and where fibrinogen levels correlate with disease activity. ${ }^{12}$ Fibrinogen levels, in addition to CRP platelet activation, have been previously demonstrated to be associated with worse outcomes following aSAH. ${ }^{10}$ Although we have no evidence that IL-1Ra improves outcome by reducing fibrinogen levels, it is nevertheless reassuring that in our study, anakinra reduces a number of inflammatory markers that have been associated with poor outcomes following aSAH.

We acknowledge limitations and shortcomings in our clinical trial. The characteristics of our study population reflected those of the disease population. Groups were similar with respect to clinical severity (WFNS and Fisher grades), the day postictus, and age. As expected, most patients were women and a high proportion were smokers. For pragmatic reasons, this was an open-label, single-blinded study, but the primary outcome was a laboratory analysis. All laboratory staff were blinded to treatment allocation, and IL-1Ra assays were performed after measurement and documentation of other cytokines. Our block sampling technique introduced additional variability within groups due to timing. However, this additional variability was balanced by design, and we observed clear differences despite this.

Another potential shortcoming of the study is that most of the patients assigned to the IMP group were administered anakinra on Day $2(77 \%)$ or Day $3(7 \%)$ following ictus. This is not reflective of its potential clinical use, where anakinra could be given immediately after diagnosis of $\mathrm{SAH}$, as is routine practice with nimodipine in the United Kingdom. The main reason for the delay in our study was to ensure that patients, or their representatives, had adequate time to consider the information they were provided prior to giving their consent.

There is increasing preclinical ${ }^{5}$ and clinical evidence ${ }^{25}$ suggesting that IL-1 is associated with increasing blood- 
brain barrier permeability and worsening clinical outcome. Therefore, IL-1Ra could have potentially significant benefit if given earlier following aSAH. Similarly, the beneficial effect of anakinra on limiting neurotoxicity and hememediated inflammation may be significantly enhanced with earlier administration. For this reason, we intend to adopt ultra-early administration of the drug, i.e., as soon as aSAH is diagnosed, in our planned Phase III study.

Anakinra is currently licensed for use as a first-line therapy for neonatal-onset multisystem inflammatory disease and as a second-line treatment for adult rheumatoid arthritis (www.kineretrx.com). In these patients, for whom it is given as a long-term treatment, anakinra has an excellent safety and tolerability profile. ${ }^{31}$ Our group has used short-term (4-72 hours) anakinra treatment in patients with stroke and SAH, using both IV and SC formulations, with a similar favorable safety profile. .7, $, 11,32$ To our knowledge, this is the first reported study in which SC anakinra was administered for longer periods (up to 21 days) in this population cohort and for which there is a plasma inflammatory profile extending beyond 72 hours. Moreover, to ensure minimal peak-trough fluctuations, our dosing regimen was twice rather than once daily.

Most participants (81\%) receiving anakinra had a relatively good WFNS grade (WFNS Grades I-III) with a good level of consciousness, and they tolerated the drug well. No safety concerns were experienced throughout the duration of the study. Despite the fact that people with aSAH have a high incidence of infection (particularly hospital-acquired pneumonia and ventriculitis), there was no statistically significant difference in the proportion of patients reported to have infection (21 infections in the actively treated group and 25 in the control group). More of the infections reported as SAEs were in the actively treated group, but this did not achieve statistical significance. There was no trend to any particular infection type.

There have been many Phase III clinical studies in SAH, but only the British Aneurysm Nimodipine Trial has demonstrated efficacy. ${ }^{1}$ Oral nimodipine demonstrated a significant reduction in cerebral infarction and poor outcome at 3 months by reducing the number of deaths and disabilities in patients with episodes of DCI. Although the causes for DCI are multifold, studies suggest that nimodipine acts primarily by reversing macroscopic cerebral artery vasospasm. ${ }^{2}$ Phase III studies further targeting arterial spasm (in part or wholly), including magnesium and clazosentan, have failed to show additional benefit on outcome. Given the effect size of nimodipine, any further improvement in outcome following SAH is more likely to be obtained by targeting causes of DCI and neurodegeneration other than arterial spasm.

The theoretical multimodal mechanism of action of statins (including the potential effect of statins on inflammation) was promising; however, both low- and high-dose simvastatin failed to show an adequate suppression of inflammation (CRP) and improved outcome. ${ }^{21}$ The causes of failure for these Phase III studies are varied and include insufficient pharmacokinetic information, lack of proof of action in the target disease population, alteration of beneficial physiological responses, as well as others. The enthusiasm of neurosurgical centers worldwide to continue to recruit patients to Phase III studies in aSAH reflects the appreciation that research into improving outcomes in this patient group remains a significant unmet clinical demand.

Current strategies being considered besides IL-1 antagonism include reducing oxidative stress ${ }^{26}$ and endothelin expression..$^{35}$ To our knowledge, this study is the first to show a significant reduction in plasma inflammation following aSAH, and therefore places IL-1 antagonism as a promising strategy for future Phase III studies. Our study was not powered to compare clinical outcomes between the active and control groups; however, it provides crucial pilot data for a Phase III study, for which funding is currently being sought.

\section{Conclusions}

We have studied the effects of IL-1 and its antagonist at the cellular level in experimental models of cerebrovascular disease. We have demonstrated proof of biological efficacy of IL-1 antagonism at both the preclinical and clinical levels using different administration routes. In conjunction with previously published data, this study provides robust pharmacokinetic data for SC anakinra with respect to achieving therapeutic plasma concentrations in patients with aSAH. We now have evidence that 1) IL-1 is detrimental in cerebral ischemia; 2) IL-6 and CRP are associated with poor outcome after aSAH; and 3) SC anakinra, when given within a reasonable time window after $\mathrm{SAH}$, is associated with a reduction in IL-6 plasma levels. With these data, anakinra is a very promising candidate for neuroprotection after SAH and is uniquely positioned for evaluation in a Phase III study.

\section{Acknowledgments}

We acknowledge the neurosurgical staff at the Hyperacute Stroke Research Centre at Salford Royal NHS Foundation Trust (Victoria O'Loughlin and her team), the University Hospital North Midlands (Holly Maguire and her team), and the Walton Clinical Trials Unit (Dave Watling and his team) for support with patient recruitment and interventions. Plasma analyses were performed by the team at the Biomedicine Laboratories at Salford Royal NHS Foundation Trust. We are grateful to the neurosurgical teams at all 3 centers for their support of this study, as well as to the patients who participated. The study was supported by the UK Medical Research Council (EudraCT: 2011-001855-35). The study was funded by a Medical Research Council Developmental Pathway and Clinical Studies Grant (G1001252; $\leq 700,000)$. Pippa Tyrrell, Andrew King, Stephen Hopkins, Andrew Vail, James Galea, Sharon Hulme, and Sylvia Scarth were supported by the grant. All consumables, equipment, and estate costs were supported by the Medical Research Council grant. Prof. Dame N. Rothwell is a nonexecutive director of AstraZeneca, which has no financial or other interest in the drug used in this study.

\section{References}

1. Adamczyk P, He S, Amar AP, Mack WJ: Medical management of cerebral vasospasm following aneurysmal subarachnoid hemorrhage: a review of current and emerging therapeutic interventions. Neurol Res Int 2013:462491, 2013

2. Allen GS, Ahn HS, Preziosi TJ, Battye R, Boone SC, Boone $\mathrm{SC}$, et al: Cerebral arterial spasm - a controlled trial of nimodipine in patients with subarachnoid hemorrhage. N Engl J Med 308:619-624, 1983 
3. Arend WP, Welgus HG, Thompson RC, Eisenberg SP: Biological properties of recombinant human monocyte-derived interleukin 1 receptor antagonist. J Clin Invest 85:16941697, 1990

4. Banwell V, Sena ES, Macleod MR: Systematic review and stratified meta-analysis of the efficacy of interleukin-1 receptor antagonist in animal models of stroke. J Stroke Cerebrovasc Dis 18:269-276, 2009

5. Blamire AM, Anthony DC, Rajagopalan B, Sibson NR, Perry $\mathrm{VH}$, Styles P: Interleukin-1 $\beta$-induced changes in blood-brain barrier permeability, apparent diffusion coefficient, and cerebral blood volume in the rat brain: a magnetic resonance study. J Neurosci 20:8153-8159, 2000

6. Clark SR, McMahon CJ, Gueorguieva I, Rowland M, Scarth $\mathrm{S}$, Georgiou R, et al: Interleukin-1 receptor antagonist penetrates human brain at experimentally therapeutic concentrations. J Cereb Blood Flow Metab 28:387-394, 2008

7. Emsley HC, Smith CJ, Georgiou RF, Vail A, Hopkins SJ, Rothwell NJ, et al: A randomised phase II study of interleukin-1 receptor antagonist in acute stroke patients. J Neurol Neurosurg Psychiatry 76:1366-1372, 2005

8. Feigin VL, Lawes CM, Bennett DA, Barker-Collo SL, Parag $\mathrm{V}$ : Worldwide stroke incidence and early case fatality reported in 56 population-based studies: a systematic review. Lancet Neurol 8:355-369, 2009

9. Fisher CM, Kistler JP, Davis JM: Relation of cerebral vasospasm to subarachnoid hemorrhage visualized by computerized tomographic scanning. Neurosurgery 6:1-9, 1980

10. Frontera JA, Aledort L, Gordon E, Egorova N, Moyle H, Patel A, et al: Early platelet activation, inflammation and acute brain injury after a subarachnoid hemorrhage: a pilot study. J Thromb Haemost 10:711-713, 2012

11. Galea J, Ogungbenro K, Hulme S, Greenhalgh A, Aarons L, Scarth S, et al: Intravenous anakinra can achieve experimentally effective concentrations in the central nervous system within a therapeutic time window: results of a dose-ranging study. J Cereb Blood Flow Metab 31:439-447, 2011

12. Gilliam BE, Reed MR, Chauhan AK, Dehlendorf AB, Moore TL: Evidence of fibrinogen as a target of citrullination in IgM rheumatoid factor-positive polyarticular juvenile idiopathic arthritis. Pediatr Rheumatol Online J 9:8, 2011

13. Greenhalgh AD, Brough D, Robinson EM, Girard S, Rothwell NJ, Allan SM: Interleukin-1 receptor antagonist is beneficial after subarachnoid haemorrhage in rat by blocking haem-driven inflammatory pathology. Dis Model Mech 5:823-833, 2012

14. Gueorguieva I, Clark SR, McMahon CJ, Scarth S, Rothwell NJ, Tyrrell PJ, et al: Pharmacokinetic modelling of interleukin-1 receptor antagonist in plasma and cerebrospinal fluid of patients following subarachnoid haemorrhage. Br J Clin Pharmacol 65:317-325, 2008 (Erratum in BR J Clin Pharmacol 66:754, 2008)

15. Gueorguieva I, Ogungbenro K, Graham G, Glatt S, Aarons L: A program for individual and population optimal design for univariate and multivariate response pharmacokinetic-pharmacodynamic models. Comput Methods Programs Biomed 86:51-61, 2007

16. Helbok R, Schiefecker AJ, Beer R, Dietmann A, Antunes AP, Sohm F, et al: Early brain injury after aneurysmal subarachnoid hemorrhage: a multimodal neuromonitoring study. Crit Care 19:75, 2015

17. Helmy A, Guilfoyle MR, Carpenter KLH, Pickard JD, Menon DK, Hutchinson PJ: Recombinant human interleukin-1 receptor antagonist in severe traumatic brain injury: a phase II randomized control trial. J Cereb Blood Flow Metab 34:845-851, 2014

18. Hendryk S, Jarzab B, Josko J: Increase of the IL-1 $\beta$ and IL-6 levels in CSF in patients with vasospasm following aneurysmal SAH. Neuroendocrinol Lett 25:141-147, 2004
19. Jedrzejowska-Szypułka H, Larysz-Brysz M, Kukla M, Snietura M, Lewin-Kowalik J: Neutralization of interleukin-1 $\beta$ reduces vasospasm and alters cerebral blood vessel density following experimental subarachnoid hemorrhage in rats. Curr Neurovasc Res 6:95-103, 2009

20. Kao HW, Lee KW, Kuo CL, Huang CS, Tseng WM, Liu CS, et al: Interleukin-6 as a prognostic biomarker in ruptured intracranial aneurysms. PLoS One 10:e0132115, 2015

21. Kirkpatrick PJ, Turner CL, Smith C, Hutchinson PJ, Murray GD: Simvastatin in aneurysmal subarachnoid haemorrhage (STASH): a multicentre randomised phase 3 trial. Lancet Neurol 13:666-675, 2014

22. Kostulas N, Kivisäkk P, Huang Y, Matusevicius D, Kostulas $\mathrm{V}$, Link $\mathrm{H}$ : Ischemic stroke is associated with a systemic increase of blood mononuclear cells expressing interleukin-8 mRNA. Stroke 29:462-466, 1998

23. McColl BW, Rothwell NJ, Allan SM: Systemic inflammatory stimulus potentiates the acute phase and CXC chemokine responses to experimental stroke and exacerbates brain damage via interleukin-1- and neutrophil-dependent mechanisms. J Neurosci 27:4403-4412, 2007

24. McMahon CJ, Hopkins S, Vail A, King AT, Smith D, Illingworth KJ, et al: Inflammation as a predictor for delayed cerebral ischemia after aneurysmal subarachnoid haemorrhage. J Neurointerv Surg 5:512-517, 2013

25. Michael BD, Griffiths MJ, Granerod J, Brown D, Keir G, Wnęk G, et al: The interleukin-1 balance during encephalitis is associated with clinical severity, blood-brain barrier permeability, neuroimaging changes, and disease outcome. J Infect Dis 213:1651-1660, 2016

26. Munakata A, Ohkuma H, Nakano T, Shimamura N, Asano K, Naraoka M: Effect of a free radical scavenger, edaravone, in the treatment of patients with aneurysmal subarachnoid hemorrhage. Neurosurgery 64:423-429, 2009

27. Osuka K, Suzuki Y, Tanazawa T, Hattori K, Yamamoto N, Takayasu M, et al: Interleukin-6 and development of vasospasm after subarachnoid haemorrhage. Acta Neurochir (Wien) 140:943-951, 1998

28. Rinkel GJE, Algra A: Long-term outcomes of patients with aneurysmal subarachnoid haemorrhage. Lancet Neurol 10:349-356, 2011

29. Robson RL, Westwick J, Brown Z: Interleukin-1-induced IL-8 and IL-6 gene expression and production in human mesangial cells is differentially regulated by cAMP. Kidney Int 48:1767-1777, 1995

30. Sarrafzadeh A, Schlenk F, Gericke C, Vajkoczy P: Relevance of cerebral interleukin-6 after aneurysmal subarachnoid hemorrhage. Neurocrit Care 13:339-346, 2010

31. Schiff MH, DiVittorio G, Tesser J, Fleischmann R, Schechtman J, Hartman S, et al: The safety of anakinra in high-risk patients with active rheumatoid arthritis: six-month observations of patients with comorbid conditions. Arthritis Rheum 50:1752-1760, 2004

32. Singh N, Hopkins SJ, Hulme S, Galea JP, Hoadley M, Vail A, et al: The effect of intravenous interleukin-1 receptor antagonist on inflammatory mediators in cerebrospinal fluid after subarachnoid haemorrhage: a phase II randomised controlled trial. J Neuroinflammation 11:1, 2014

33. Teasdale GM, Drake CG, Hunt W, Kassell N, Sano K, Pertuiset B, et al: A universal subarachnoid hemorrhage scale: report of a committee of the World Federation of Neurosurgical Societies. J Neurol Neurosurg Psychiatry 51:1457, 1988

34. Thornton P, Pinteaux E, Gibson RM, Allan SM, Rothwell NJ: Interleukin-1-induced neurotoxicity is mediated by glia and requires caspase activation and free radical release. J Neurochem 98:258-266, 2006

35. Tseng MY, Hutchinson PJ, Richards HK, Czosnyka M, Pickard JD, Erber WN, et al: Acute systemic erythropoietin therapy to reduce delayed ischemic deficits following aneurysmal 
subarachnoid hemorrhage: a Phase II randomized, doubleblind, placebo-controlled trial. Clinical article. J Neurosurg 111:171-180, 2009

36. Turner CL, Budohoski K, Smith C, Hutchinson PJ, Kirkpatrick PJ, Murray GD: Elevated baseline C-reactive protein as a predictor of outcome after aneurysmal subarachnoid hemorrhage: data from the Simvastatin in Aneurysmal Subarachnoid Hemorrhage (STASH) trial. Neurosurgery 77:786-793, 2015

37. Wu W, Guan Y, Zhao G, Fu XJ, Guo TZ, Liu YT, et al: Elevated IL- 6 and TNF- $\alpha$ levels in cerebrospinal fluid of subarachnoid hemorrhage patients. Mol Neurobiol 53:32773285,2016

\section{Disclosures}

Prof. Vail, an employee of University of Manchester, received academic credit for a successful funding application to Medical Research Council.

\section{Author Contributions}

Conception and design: Galea, Ogungbenro, Hulme, Hopkins,
Vail, Rothwell, Tyrrell. Acquisition of data: Galea, Hulme, Scarth, Hoadley, Illingworth, McMahon, Tzerakis, Tyrrell. Analysis and interpretation of data: Galea, Ogungbenro, Hulme, Scarth, Hoadley, Illingworth, Hopkins, Vail, Tyrrell. Drafting the article: Galea. Critically revising the article: Galea, Ogungbenro, Hulme, Patel, Scarth, Hoadley, Illingworth, McMahon, Tzerakis, Hopkins, Vail, Rothwell, Tyrrell. Reviewed submitted version of manuscript: Galea, Hulme, Vail, Tyrrell. Approved the final version of the manuscript on behalf of all authors: Galea. Statistical analysis: Galea, Ogungbenro, Hopkins, Vail, Tyrrell. Administrative/technical/material support: Galea, Ogungbenro, Hulme, King, Rothwell, Tyrrell. Study supervision: Galea, Tyrrell.

\section{Supplemental Information}

\section{Previous Presentations}

This study was presented at the AANS/CNS Joint Cerebrovascular Section Annual Meeting, Universal City, California, February 15-16, 2016.

\section{Correspondence}

James Galea, Department of Neurosurgery, Ninewells Hospital and Medical School, University of Dundee, Dundee, DD1 9SY, United Kingdom. email: jgalea@dundee.ac.uk. 